\title{
Anti-atherosclerotic Effects of Eurycoma Longifolia (Tongkat Ali) in Rats Fed on High-fat Diet
}

Fakhria Al-Joufi ${ }^{a}$, Anil K. Saxena ${ }^{a}$, Imad M. Al-Ani ${ }^{a *}$, Norlelawati A. Talib ${ }^{a}$, Rafidah H. Mokhtar ${ }^{b}$, Norsidah Ku -Zaifah ${ }^{\mathrm{a}}$

a Department of Basic Medical Sciences, Kulliyyah of Medicine, International Islamic University Malaysia

${ }^{b}$ Faculty of Medicine, Universiti Sains Islam Malaysia, Nilai, Negeri Sembilan, Malaysia

\begin{abstract}
Atherosclerosis in cardiovascular disease (CVD) is a growing health problem, especially in developing countries. Hyperlipidemia is known as a dominant risk factor for the development of atherosclerosis. This study was designed to investigate the effects of Eurycoma Longifolia (EL) also known as Malaysian Ginseng/ Tongkat Ali on the testosterone level, biochemical changes of lipid profile and intima media thickness (IMT) in rats fed on high-fat diet. Twenty young, adult male Sprague-Dawley (SD) rats were housed for 12 weeks. After one week of acclimatization, they were randomly divided into four groups of 5 animals each and treated for 12 weeks as follow: Group ND was given only normal diet, group NDEL was given normal diet and EL extracts $(15 \mathrm{mg} / \mathrm{kg})$ dissolved in distilled water, group HFD was given only high fat diet and group HFDEL was given high fat diet and EL extracts $(15 \mathrm{mg} / \mathrm{kg})$. Rats which were treated with EL (NDEL and HFDEL) showed a significant increase $(p<0.05)$ in the testosterone levels. There was a significant decrease $(p<0.05)$ in triglyceride (TG) in HFDEL group compered to HFD group. The histological sections of aortas revealed a significant decrease $(p<0.05)$ in IMT in HFDEL as compared with HFD group. No histological changes were observed in NDEL group compared with ND group and there was no significant difference in IMT values between NDEL and ND. These findings suggest that EL is a promising protective agent against atherosclerosis induced by high-fat diet.
\end{abstract}

Keywords: Atherosclerosis, cardiovascular diseases, Eurycoma longifolia (Tongkat Ali),, hyperlipidemia, intima media thickness, testosterone.

\section{INTRODUCTION}

Atherosclerosis is a progressive inflammatory disorder of the arterial wall that is characterized by focal lipid-rich of atheroma. ${ }^{1}$ It is a growing health problem all over the world including developing countries like Malaysia, as the number of cardiovascular death of ischemic heart disease cases in Malaysia as reported by Ministry of Health $(\mathrm{MOH})$ has increased from 2556 in 2000 to 2948 in 2005. ${ }^{2}$

Atherosclerosis is initiated by endothelial cells activation and dysfunction subsequently leading to increase in the permeability of sub-endothelial layer to plasma lipids as well as increasing the adhesion of monocytes to vascular cell adhesive molecules "VCAM- 1 ". ${ }^{3}$ Monocytes differentiate into foam cells to form fatty streaks accompanied by proliferation of vascular smooth muscle cells (VSMC) leading to intimal thickening. Then, the extracellular lipid core progresses into mature atherosclerotic plaques

Corresponding author:

Imad M. AL-Ani.

Department of Basic Medical Sciences,

Kulliyyah of Medicine,

International Islamic University Malaysia.

Phone: 006017-9776014.

E-mail: imad_alani@yahoo.com resulting in narrowing of the arterial lumen leading to subsequent decrease in blood flow through the affected vessel. ${ }^{4}$ Hyperlipidemia is considered as a risk factor of developing the manifestations of atherosclerosis and its complications. ${ }^{5}$ High-fat (HF) diet model has been widely used to study disease etiology. It provides an effective means for investigating the disease mechanisms of atherosclerosis. ${ }^{6}$

Testosterone, one of the most important androgens, is now established as independent risk factor for the development of CVDs including atherosclerosis. 7 It is associated with raised fibrinogen and hypercoagulable states, enhancing atherosclerosis and atherosclerotic plaque instability and thus acute coronary syndromes. ${ }^{8}$ Testosterone may affect the development of CVD by modulating risk factors such as diabetes, insulin resistance, obesity, hypercholesterolemia, and hypertriglyceridemia. The vasculature system is a target of testosterone effect and recent evidence suggests that the testosterone hormone plays a beneficial role in cardiovascular system (CVS) particularly in endothelial regeneration. ${ }^{10}$

Although there is a significant advancement in the synthetic drugs, plants still constitute the major natural source in the medicinal system. One of the most attractive Asian herbs is Eurycoma Longifolia 
(EL). It is also known as Malaysian Ginseng/Tongkat Ali. It has long been used as traditional remedies and in folklore remedy for the treatment of many diseases in South-East Asia countries. EL is rich in various phytochemical compounds such as alkaloids and quassinoids. ${ }^{11}$ Animal studies have reported that EL has the ability to increase the testosterone levels and reverses the inhibitory effects of estrogen on testosterone synthesis. ${ }^{12}$

The supplementation of EL to androgen-deficient osteoporosis animal model has the potential to combat androgen-deficient osteoporosis. 13 Recently, histological study has demonstrated that administration of aqueous extract of EL significantly attenuated the formation of atherosclerotic plaques in aorta and preserved the vascular structure in rats fed high-fat diet. ${ }^{14}$ However, to date, the main mechanism of EL to enhance the testosterone level in circulation is poorly understood and requires further investigation. Therefore, the aims of this study were to assess the effects of $E L$ on atherosclerosis-related parameters, blood pressure, testosterone and gene-expressions in rats fed highfat diet.

\section{MATERIALS AND METHODS}

\section{Animals}

Twenty young, adult male Sprague-Dawley (SD) rats weighing 250-350 gm each were used in this study. All animals were housed for 12 weeks and caged in standard plastic cages ( 2 rats per cage). They were maintained at room temperature $\left(22-24^{\circ} \mathrm{C}\right)$ with adequate ventilation, 12 -h light-dark cycle and about $(50 \pm 5 \%)$ humidity

After one week of acclimatization, they were randomly divided into four groups of 5 animals each and treated for 12 weeks as follow: Group ND was given only normal diet, group NDEL was given normal diet and $15 \mathrm{mg} / \mathrm{kg}$ EL aqueous extracts, group HFD was given only high fat diet and group HFDEL was given high fat diet and $15 \mathrm{mg} / \mathrm{kg}$ EL aqueous extracts. This study was approved and all procedures complied with the guidelines and recommendations of IIUM ethical committee (IIUM/519/14/4/IACUC) and Malaysian National Animal Welfare Foundation.

\section{Eurycoma Longifolia Extract}

The EL extract powder PHYSTA $®$, was obtained from Biotropics Malaysia Berhad. The aqueous extract was prepared by dissolving $15 \mathrm{mg}$ of EL extract in $10 \mathrm{ml}$ of distilled water. This allowed for simple and easy weekly dose calculations according to the rat's body weight. The preparation was kept in refrigerator at temperature of $2-8 \mathrm{C}^{\circ}$ and was removed from refrigerator 30 minutes before to allow equilibrium to the rat's body temperature.

\section{High-Fat Diet}

The HFD Pellets were purchased from MP Biomedicals, California, USA (Next Gene Scientific Sdn. Bhd). The compositions of high saturated fat diet are shown in Table 1.

\section{Collection of Blood Samples}

Blood samples were collected from all experimental groups under general anesthesia; the diethyl ether. A capillary tube was gently inserted in the orbital

Table 1: The composition of high-fat diet

\begin{tabular}{ll}
\hline INGREDIENT & AMOUNT \\
\hline Casein Purified High Nitrogen & $4000 \mathrm{gm}$. \\
DL-Methionine & $60 \mathrm{gm}$. \\
Sucrose & $6116 \mathrm{gm}$. \\
Corn Starch & $4000 \mathrm{gm}$. \\
Coconut Oil Hydrogenated & $4000 \mathrm{gm}$. \\
Alphacel, Non-Nutritive Bulk & $1000 \mathrm{gm}$. \\
DL-a-Tocopherol Powder (250 & $24 \mathrm{gm}$. \\
IU/gm.) & $800 \mathrm{gm}$. \\
\hline AIN-76 Mineral Mix & \\
\hline
\end{tabular}

Plus MP Vitamin Diet Fortification Mixture 1.2 x Normal

sinus and the required volume of blood was collected in a sterile glass tube containing gel for serum separation. After allowing the blood to clot at room temperature for 20-30 minutes the blood samples were centrifuged at $3000 \mathrm{rpm}$ for 15 minutes. The centrifuged serum was stored at $-80^{\circ} \mathrm{C}$ until the biochemical evaluation was done.

\section{Biochemical Assays}

Testosterone levels were measured in serum (nmol/ L) using BioVision's Testosterone EIA kit. It operates on the basis of competition between the hormone conjugate and the testosterone in the serum for a limited number of binding sites on the antibody coated plate. Quantitative test results were obtained by measuring and comparing the absorbance reading of the wells of the samples against the standards with a microplate reader at $450 \mathrm{~nm}$.

Serum total cholesterol and high density lipoprotein cholesterol (HDL-c) were determined using Randox Laboratory kit reagents. Serum triglyceride level was estimated using Randox Laboratory test kit. Low density lipoprotein cholesterol (LDL-c) was estimated indirectly from the measured levels of triglyceride (TG), high-density lipoprotein cholesterol (HDL-C), and total cholesterol (TC) using the Friedewald equation: $\mathrm{LDL}=\mathrm{TC}-\mathrm{HDL}-(\mathrm{TG} /$ 2.17) 


\section{Histomorphometric Study}

At the end of 12th week, the rats were kept in fasted state for 12 hours prior to anesthesia, and then sacrificed by cervical dislocation. The specimens were fixed in $10 \%$ formal saline, dehydrated with gradual series of alcohol, embedded in paraffin wax. Serial transverse sections of $(5 \mu \mathrm{m})$ thickness were prepared and the sections were stained with hematoxylin and eosin (H\&E) and Verhoeff-Van Gieson (VVG).


Figure 1: A. The distance between the lumen and the external elastic lamina is measured as intimal media thickness (IMT). B. The mean of IMT of aortas in different experimental groups. * indicates a significant difference from ND group $(p<0.05)$ and $\dagger$ indicates a significant difference from HFD group $(p<0.05)$. C. Correlation analysis; showing negative correlation between IMT with the total testosterone level in NDEL (A) and between IMT with the total testosterone in HFDEL (B). D. Serum testosterone levels of different experimental groups during the treatment period. * Significant difference between the mean of testosterone from ND. \# Significant difference between the mean of testosterone from HFD $(P<0.05)$.

\section{Statistical Analysis}

All analyses were conducted using one-way and repeated measures ANOVA. The statistical software SPSSs 22.0 was used. Post-hoc comparison (Tuky's test) was employed to assess the overall significance among groups. Correlations between total testosterone level and IMT were calculated using Pearson's correlation coefficient. A $p$ value less than 0.05 was considered statistically significant.

\section{RESULTS}

\section{Histomorphometric analysis}

IMT values are given in Table 2 . Compared to ND group, NDEL and HFDEL did not exhibit a statistical
Finally, the specimens were analyzed via microscope and Dino-Capture 2.0. The histological examination was carried out at a magnification of $\times 20$ with a light microscope. The thicknesses of the intima media of aortas were photographed and measured with Dino-Capture ${ }^{\circledR}$ 2.0. The distance between the lumen and the external elastic lamina was measured as intimal media thickness (IMT) (Fig.1.A.) Atherosclerosis was examined in a blinded manner using five cross sections from each group.
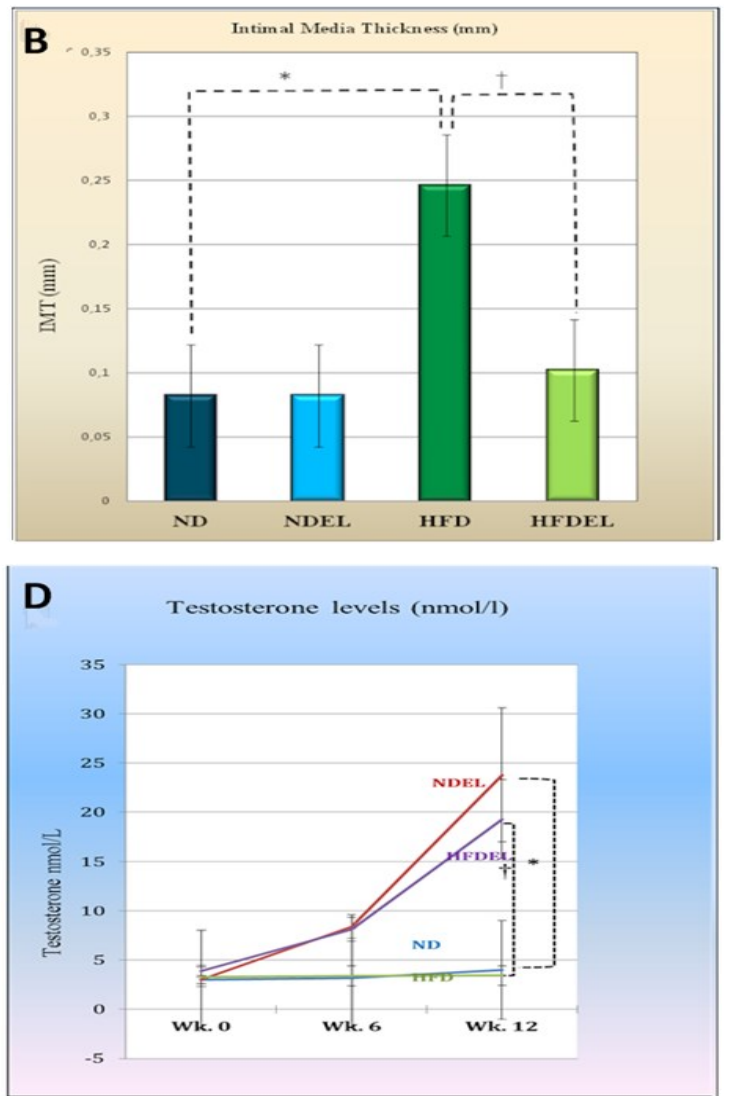
Table 2: Intima media thickness (IMT) of different groups at the end of the study

\begin{tabular}{cc}
\hline Groups & Intima media thickness \\
\hline ND & $0.082 \pm 0.005$ \\
NDEL & $0.082 \pm 0.005$ \\
HFD & $0.246 \pm 0.027^{*}$ \\
HFDEL & $0.102 \pm 0.013^{\#}$
\end{tabular}

Values are presented as means \pm SD. *Significantly $p<0.05$ ) different from ND. " Significantly $P<0.05$ ) different from HFD.

Table 3: Serum testosterone levels in all groups at Week 0,6 and 12

\begin{tabular}{lllll}
\hline Groups & ND & NDEL & HFD & HFDEL \\
Weeks & & & & \\
\hline 0 & $3 \pm 1$ & $3.02 \pm 0.4$ & $3.3 \pm 1.3$ & $3.87 \pm 0.6$ \\
6 & $3.2 \pm 0.8$ & $8.4 \pm 1.2^{*}$ & $3.4 \pm 1.02$ & $8.13 \pm 1.2^{* \#}$ \\
12 & $4 \pm 0.98$ & $23.8 \pm 6.8^{*}$ & $3.43 \pm 0.98$ & $19.3 \pm 4^{* \#}$ \\
\hline
\end{tabular}

The values are means \pm SD ( $n=5 /$ group $).{ }^{*}$ Significant difference between the mean of testosterone from ND $(\mathrm{P}<0.05)$. " Significant difference between the mean of testosterone from HFD $(P<0.05)$.
Lipid profile levels of all experimental groups were evaluated and showed in table 4 . There were highly significant increases in TC and TG in both groups (HFD and HFDEL) as compared to ND group (Figs.2.A and 2.B) On the other hand, there was no significant change $(p>0.05)$ in lipid profile parameters of NDEL when compared to ND (Figs.2.A and 2.B).

HFDEL rats have high significant decrease $(p<0.05)$ in its TG levels as compare to HFD group (Table 4 and Fig. 2. B) There were no significant differences in TC, HDL-C and LDL-C in HFDEL compared to HFD (Table 3).

Although, there was a reduction in TC and LDL-C in both treated groups, this reduction was not statistically significant $(p>0.05)$ (Figs.2.A and 2.C). The results in table 4 did not show any significant change in HDL-C between the treated groups and untreated groups $(p>0.05)$ (Fig. 2.D).

There was a tendency toward a negative correlation between total testosterone level and IMT in NDEL $(r=-0.64) \quad(p=0.25)$. In HFDEL there was a trend towards a moderate negative correlation between the total testosterone level and IMT with $r=-0.53$, although this did not reach statistical significance $(p=0.24)$ (Fig. 1.C).

Table 4: Descriptive Statistics of Total Cholesterol at Week 0, 6 and 12

\begin{tabular}{|c|c|c|c|c|c|}
\hline \multirow{2}{*}{\multicolumn{2}{|c|}{ PARAMETERS }} & \multicolumn{4}{|c|}{ GROUPS } \\
\hline & & ND & NDEL & HFD & HFDEL \\
\hline \multirow{4}{*}{ Week 0} & TC & $0.9 \pm 0.3$ & $0.8 \pm 0.4$ & $0.8 \pm 0.3$ & $0.8 \pm 0.3$ \\
\hline & TAG & $0.55 \pm 0.2$ & $0.51 \pm 0.4$ & $0.55 \pm 0.27$ & $0.59 \pm 0.28$ \\
\hline & LDL & $0.43 \pm 12$ & $0.39 \pm 0.2$ & $0.30 \pm 0.14$ & $0.38 \pm 0.2$ \\
\hline & $\mathrm{HDL}$ & $0.21 \pm 0.1$ & $0.23 \pm 0.14$ & $0.23 \pm 0.11$ & $0.18 \pm 0.1$ \\
\hline \multirow{4}{*}{ Week 6} & $\mathrm{TC}$ & $1 \pm 0.2$ & $0.9 \pm 0.2$ & $1.3 \pm 0.3$ & $1.2 \pm 0.2$ \\
\hline & TAG & $0.7 \pm(0.2$ & $0.65 \pm 0.2$ & $1.22 \pm 0.2$ & $0.84 \pm 0.23$ \\
\hline & LDL & $0.45 \pm 0.2$ & $0.39 \pm 0.2$ & $0.45 \pm 0.3$ & $0.45 \pm 0.3$ \\
\hline & $\mathrm{HDL}$ & $0.25 \pm 0.1$ & $0.23 \pm 0.1$ & $0.32 \pm 0.2$ & $0.3 \pm 0.1$ \\
\hline \multirow{4}{*}{ Week 12} & $\mathrm{TC}$ & $0.9 \pm 0.2$ & $0.55 \pm 0.3$ & $1.4 \pm 0.2^{*}$ & $1 \pm 0.3$ \\
\hline & TAG & $0.5 \pm 0.2$ & $0.25 \pm 0.1$ & $1.5 \pm 0.9^{*}$ & $0.79 \pm 0.3^{\#}$ \\
\hline & LDL & $0.44 \pm 0.2$ & $0.34 \pm 0.1$ & $0.62 \pm 0.24$ & $0.38 \pm 0.1$ \\
\hline & $\mathrm{HDL}$ & $0.21 \pm 0.1$ & $0.19 \pm 0.2$ & $0.3 \pm 0.1$ & $0.3 \pm 0.1$ \\
\hline
\end{tabular}

The values are means \pm SD ( $n=5$ /group). ${ }^{*}$ Significant difference between the mean of testosterone from ND $(P<0.05)$. Significant difference between the mean of testosterone from HFD $(P<0.05)$. 

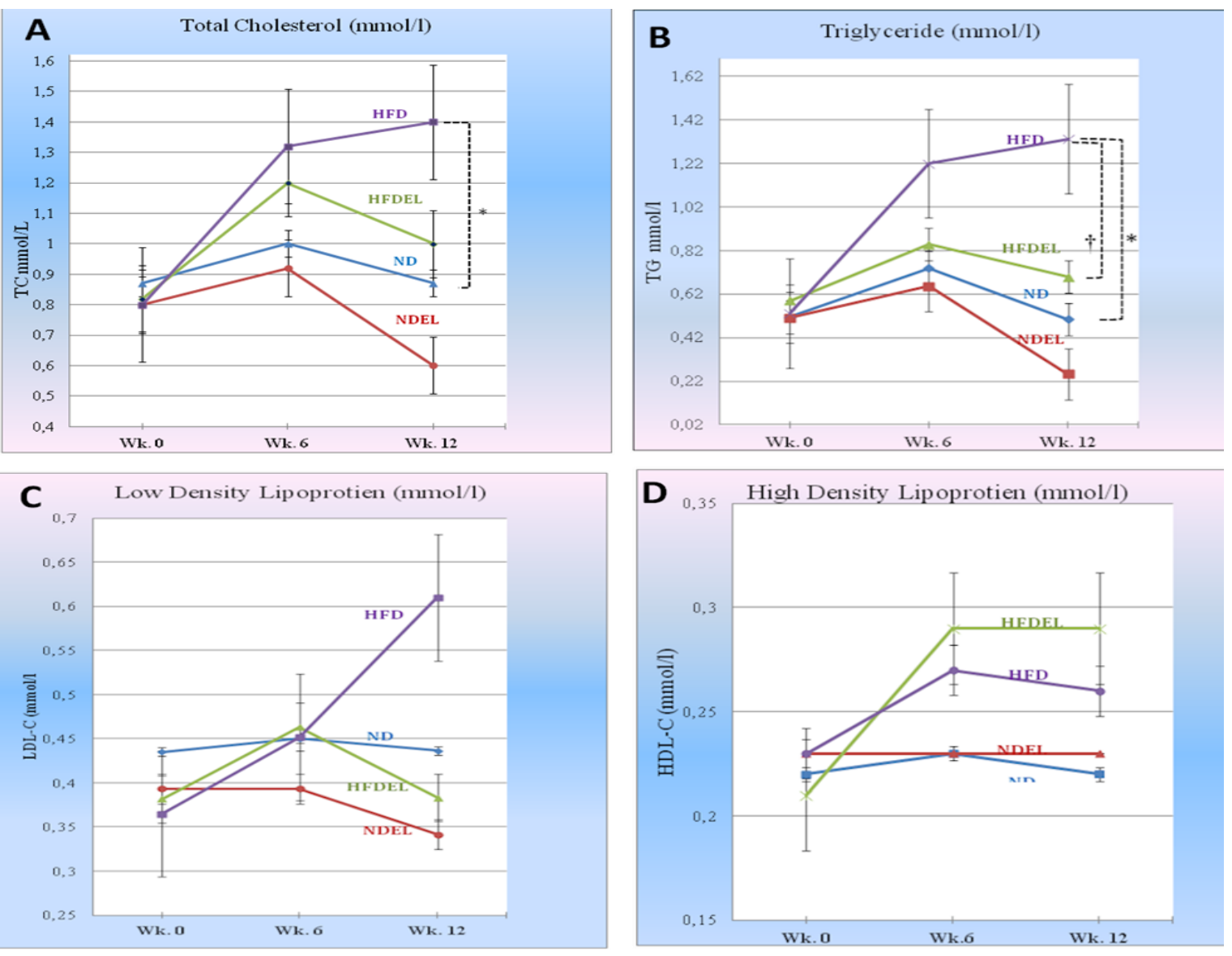

Figure 2: A. Total cholesterol (TC) of different experimental groups at wk.0, 6 \&12.* indicates a significant difference between HFD vs. ND $(P<0.05)$. B. Triglyceride $(T G)$ of different experimental groups at wk.0, $6 \& 12$. * indicates a significant difference between HFD vs. ND, \# indicates a significant difference between HFD vs. HFDEL $(P<0.05)$. C. Low density lipoprotein-cholesterol $(L D L-C)$ of different experimental groups at wk.0, 6 \&12. Notice the trend of line of LDL deceases in both treated groups. D. High density lipoprotein-cholesterol (HDL-c) of different experimental groups at wk.0, 6 \&12. Notice the trend of lines of HDL increases in both treated groups.

\section{DISCUSSION}

The high fat diet-induced atherosclerosis model in the current study was employed to produce atherosclerotic lesion in the aorta to answer the question of whether EL can prove beneficial effects for CVS. Indeed, reports from previous studies indicated that EL has various bioactive compounds. These bioactive compounds were studied extensively to enhance testosterone levels. To the best of our knowledge, no animal experiment has yet been investigated on CVS.

Twelve weeks of HFD treatment ensured adequate hyperlipidemia in the rats' blood to generate atherosclerotic plaque. Six weeks after the food and treatment interventions the treated groups (NDEL and HFDEL) displayed a considerable rise in testosterone levels in comparison with the untreated groups (ND and HFD).

Generally, these results showed that there was insignificant differences between the results of the treated groups (NDEL and FHDEL) after dosing the male rats with $15 \mathrm{mg} / \mathrm{kg}$ of $\mathrm{EL}$, and this may be attributable to the beneficial effect of EL itself but not to the food, thus providing further evidence of the folk-use of EL as aphrodisiac and testosterone booster.

This was consistent with reports from previous studies on phytoandrogenic effect of EL in rats. ${ }^{15}$ Though the aphrodisiac effect of EL has been extensively studied, the mechanisms underlying this effect remain far less clear. One of the proposed mechanisms is that EL has the ability to activate CYP17, steroid 17-alpha-monooxygenase, which catalyzes the last step of androgen biosynthesis in testes. ${ }^{16}$

In the current study, rats fed with diet rich in saturated fat showed increment in serum TC and TG in both HFD and HFDEL rats. Ample evidence has accumulated through animal studies and small clinical trials have shown that TG is associated with atherogenesis. ${ }^{17,18}$ Actually, the triglycerides can damage the arterial inner-lining and initiate the atherosclerotic plaque in aorta. ${ }^{19}$ Previous studies on hyperlipidemia has found that the elevated serum triglyceride (TG) level is correlated with generation of small dense LDLs "pattern B". 20, 21 
There is strong evidence available suggesting a relationship between LDL size and hypertriglyceridemia. ${ }^{22,23}$ Actually, there are two patterns of LDL based on its size; a normal LDL pattern (pattern A with LDL size > $25.5 \mathrm{~nm}$ ) and a small dense LDL (pattern $B$ with $L D L$ size $<25.5 \mathrm{~nm}$ ). A small dense $L D L$ is responsible for oxidation with an increased risk of atherosclerosis. Furthermore, they have poor binding affinity to LDL-receptors. ${ }^{24}$ By that characteristic the small dense LDL particles can easily penetrate the gaps between endothelium cells to sub-endothelium region. Thus, there is a possibility that the daily oral administration of EL appeared to reduce triglyceride levels by inhibiting triglyceride synthesis, in which a reduction in LDL's size might occurred. The serum HDL-cholesterols of all the experimental groups did not show significant $(p>0.05)$ change.

Also the TC and LDL-cholesterols did not change significantly $(p>0.05)$. In fact, several studies have consistently shown correlation between serum level of TC, TG, HDL-cholesterols and LDL-cholesterols with atherosclerosis. ${ }^{25}, 26$ It may therefore be that the action of EL in reducing atherosclerotic lesion may be due to phyto-androgenic effect of EL.

The bioactive peptide $(4.3 \mathrm{kDa})$ isolated from EL has potential phytoandrogenic properties and proved to improve sexual health and has significant positive effects as diabetes and stress reducer. ${ }^{15}$ Phytochemical studies revealed that EL has anti-ulcer, anti-cancer, anti-stress, antiparasite, anti-tumor properties and has potential to treat various diseases and to replace the current treatment. ${ }^{27}$

The present histomorphometric study, demonstrated that the IMT measurements revealed no significant differences among NDEL and HFDEL groups compared to ND, while there was a remarkable difference between HFD compared to ND. The treated (HFDEL) group revealed comparable thickness to that of ND group denoting attenuation of atherosclerotic lesion induced by high-fat diet with a clearly significant difference from untreated (HFD) group indicating that EL has a significant hypolipidemic effect. The possible explanation to this finding could be due to the hypolipidemic effect of EL on decreasing triglyceride level which plays a vital role in atherogenesis. This explanation comes in agreement with previous report that demonstrated hypolipidemic effect of Piper betel in high fat diet treated rats. ${ }^{19}$

\section{CONCLUSIONS}

The results of the current study demonstrated that El has the potential to be used as testosterone booster. In addition, EL is potentially protective against atherosclerosis through the reduction of triglyceride in serum of rats fed the high-fat diet showing promising endeavor in managing atherosclerosis induced by HF diet in future.

\section{ACKNOWLEDGEMENT}

Authors would like to thank IIUM Research Management Center and USIM for sponsoring and supporting this research study (grant \# B13-0680953). We warmly thank all the staff of pharmacology lab and Molecular Research Laboratory department for their help and skillful technical assistance.

\section{Conflict of interest statement}

The authors declare that they have no personal or financial conflict of interest.

\section{REFERENCES}

1. Walker BR, Colledge NR, Ralston SH, et al. Davidson's Principles and Practice of Medicine, $22^{\text {nd }}$ Edition. 2014; Churchill Livingstone. ISBN: 978-0-7020-5035-0.

2. Muda Z, Abdul Kadir A, Yusof Z, et al. Premature Coronary Artery Disease among Angiographically Proven Atherosclerotic Coronary Artery Disease in North East of Peninsular Malaysia. International J Collaborative Res Internal Medicine \& Public Health 2013; 5: 507-16.

3. Badimon L, Padró T, Vilahur G. Atherosclerosis, platelets and thrombosis in acute ischaemic heart disease. European Heart Journal: Acute Cardiovascular Care 2012; 1: 60-7.

4. Ziqiang $X$, Jingjun $W$, Jianjian Z, et al. Tadalafil attenuates graft arteriosclerosis of aortic transplant in a rat model. Iran J Basic Med Sci 2015; 18: 927-31.

5. Park SH, Sung YY, Nho KJ, et al. Protective activity ethanol extract of the fruits of Illicium verum against atherogenesis in apolipoprotein $\mathrm{E}$ knockout mice. Complementary and Alternative Medicine 2015;15: 232. doi: 10.1186/s12906-015 -0750-0

6. Rong A, Borjihan G, Qiao YJ. Effect of GBOT on blood lipid and blood glucose metabolism in rats with atherosclerosis. Genet Mol Res 2015; 14: 7801-10.

7. Daka B, Langer RD, Larsson CA, et al. Low concentrations of serum testosterone predict acute myocardial infarction in men with type 2 diabetes mellitus. Endocr Disord 2015; 15: 35. doi: 10.1186/s12902-015-0034-1.

8. Morris PU, Channer KS. Testosterone and cardiovascular disease in men. Asian J Andrology 2012; 14: 428-35.

9. Muller M, Schouw YT, Thijssen et al. Endogenous Sex Hormones and Cardiovascular Disease in Men. J Clin Endocrinol Metab 2003; 88: 5076-86.

10. Maggio M, Basaria S. Welcoming low testosterone as a cardiovascular risk factor. International Journal of Impotence Research 2009; 21: 261-4.

11. Tong KL, Chan KL, AbuBakar S, et al. The In Vitro and In Vivo Anti-Cancer Activities of a Standardized Quassinoids Composition from 
Eurycoma longifolia on LNCaP Human Prostate Cancer Cells. Plos One 2015; DOI:10.1371/ journal.pone.0121752

12. Wahab NA, Mokhtar NM, Halim WN, et al. The Effect of Eurycoma Longifolia Jack on Spermatogenesis in Estrogen-Treated Rats. Clinic 2010; 65: 93-8.

13. Abdul Razak SH, Shuid AN, Mohamed IN. Combined Effects of Eurycoma longifolia and Testosterone on Androgen-Deficient Osteoporosis in a Male Rat Model. EvidenceBased Complementary and Alternative Medicine 2012; 6. doi: 10.1155/2012/872406

14. Al-Joufi F, Al-Ani IM, Saxena AK, et al. Assessment of Anti-Atherosclerotic effect of Eurycoma longifolia Extract on high-fat diet model in rats. I: Histological Study. European J Anatomy 2016; 20 (2): 131-36.

15. George A, Henkel R. Phytoandrogenic properties of Eurycoma longifolia as natural alternative to testosterone replacement therapy. Andrologia 2014; 46: 708-21.

16. Tajul Ariff AS, Soelaiman IN, Pramanik J, et al. Effects of Eurycoma longifolia on Testosterone Level and Bone Structure in an Aged Orchidectomised Rat Model. Evidencebased Complementary and Alternative Medicine : eCAM 2012; 818072. doi: $10.1155 / 2012 / 818072$

17. McCormick SPA. Lipoprotein (a): Biology and Clinical Importance. Clin Biochem Rev 2004; 25: 69-80.

18. Correa CS, Teixeira BC, Bittencourt A. Postprandial lipemia and cardiovascular diseases: the beneficial role of strength exercise. J Vasc Bras 2014; 13: 123-30.

19. Thirumalai T, Tamilselvan N, David E. Hypolipidemic activity of Piper betel in high fat diet induced hyperlipidemic rat. Journal of Acute Disease 2014; 3: 131-5.

20. Sharma RK, Singh VN, Reddy HK. Thinking beyond low-density lipoprotein cholesterol: strategies to further reduce cardiovascular risk. Vas Health Risk Manag 2009; 5:793-9.

21. Manjunath CN, Rawal JR, Irani PM, etal. Atherogenic dyslipidemia. Indian J Endocrinol Metab 2013; 17: 969-76.

22. Carmena R, Duriez P, Fruchart J-C. Atherogenic Lipoprotein Particles in Atherosclerosis. Circulation 2004; 109: III-2-III7.

23. Tchernof A, Després J. Pathophysiology of Human Visceral Obesity: An Update. Physiological Reviews 2013; 93: 359-404

24. Kondo A, Muranaka Y, Ohta I, et al. Relationship between triglyceride concentrations and LDL size evaluated by malondialdehyde-modified LDL. Clin Chem 2013; 47: 893-900.

25. Vergeer M, Holleboom AG, Kastelein JP, et al. The HDL hypothesis: does high-density lipoprotein protect from atherosclerosis? J Lipid Res 2010; 51: 2058-73.
26. Yamagishi $\mathrm{K}$, Iso $\mathrm{H}$, Yatsuya $\mathrm{H}$, et al. Dietary intake of saturated fatty acids and mortality from cardiovascular disease in Japanese. Am J Clin Nutr 2010; 92: 759-65.

27. Mohamed AN, Vejayan J, Yusoff MM. Review on Eurycoma longifolia Pharmacological and Phytochemical Properties. Journal of Applied Sciences 2015; 15: 831-44. 
